\title{
Maximização da Razão de Capacidade em sistemas WCDMA aplicando Algoritmo Genético e Simmulated Annealing
}

\author{
Sebastián Espinosa Rueda e Paulo Roberto Rosa Lopes Nunes
}

\begin{abstract}
Resumo- O nível de potência do Canal Piloto Comum (CPICH) do padrão WCDMA é uma ferramenta importante para conseguir um equilíbrio entre a cobertura e a capacidade dos serviços de voz e dados oferecidos num sistema UMTS. Define-se a razão de capacidade num Nó-B, como a razão entre a potência disponível para os canais de tráfego e a demanda total de tráfego para servir aos usuários dentro da área de cobertura. O objetivo deste trabalho é maximizar a razão de capacidade, minimizando o número de usuários sem cobertura. O problema resultante é multi-objetivo e técnicas de otimização numérica como o Algoritmo Genético (AG) e o Recozimento Simulado (Simmulated Annealing - SA) podem oferecer soluções válidas para este problema, dependendo de uma adequada configuração da heurística. Dados reais de uma rede UMTS na cidade de Lisboa são empregados nas simulações computacionais. As duas heurísticas conseguiram incrementar a capacidade média oferecida do sistema, minimizando a quantidade de usuários sem cobertura. Porém, a solução proposta pelo AG mostrou-se como a mais adequada para ser implementada.
\end{abstract}

Palavras-Chave- Algoritmos Genéticos, Simmulated Annealing, WCDMA, Otimização, Problema Multi-Objetivo.

\begin{abstract}
The power level of the Common Pilot Channel (CPICH) of the WCDMA standard is an important feature to get an equilibrium between coverage and capacity of the voice and data services offered by an UMTS cellular system. The capacity ratio in a Node- $B$ is defined as the ratio between the power devoted to traffic channels and the total power needed to serve the users inside the coverage area. The main objective of this article is to maximize the capacity ratio, minimizing the number of non-coverage users. The problem is multi-objective and numerical optimization methods such as Genetic Algorithm (GA) and Simmulated Annealing (SA) can offer valid solutions to this problem if they have the right configurations. Real-life data of a WCDMA network in Lisbon are used in the computational simulations. Both heuristics increased the average system capacity reducing the quantity of non-coverage users to a minimum. However, the solution proposed by the GA is the most fitted to be deployed.
\end{abstract}

Keywords- Genetic Algorithms, Simmulated Annealing, WCDMA, Optimization, Multi-Objetive Problem.

Sebastián Espinosa Rueda e Paulo Roberto Rosa Lopes Nunes, Seção de Engenharia Elétrica, Instituto Militar de Engenharia - IME, Praça General Tibúrcio 80, Praia Vermelha, 22290-270, Rio de Janeiro - RJ, Brasil. Emails: sebas_espinosa@yahoo.com, prnunes@ime.eb.br.

Os autores agradecem ao Laboratório de Comunicações Digitais do IME pelas facilidades oferecidas para a realização do presente trabalho.

\section{INTRODUÇÃO}

As redes de telefonia celular de terceira geração (3G) UMTS estão já sendo implementadas e se espera um crescimento da demanda dos usuários pelos serviços de dados que serão oferecidos pelas operadoras. A interface aérea é dada pelo padrão WCDMA (Wideband Code Division Multiple Access). O nível de potência do Canal Piloto Comum (Common Pilot Channel), CPICH é uma ferramenta efetiva para o controle da cobertura e da capacidade oferecida por uma estação Nó-B (ERB) aos usuários. Num sistema limitado por interferência o valor adequado do canal CPICH em cada Nó-B permite encontrar o equilíbrio necessário entre cobertura e capacidade [1]. Portanto, considerando um sistema já operativo é necessária a otimização permanente deste parâmetro. O projeto MOMENTUM [2], finalizado em 2003, disponibilizou informações completas sobre redes UMTS operativas na Europa com o objetivo de que novas pesquisas possam ser realizadas sem depender exclusivamente das informações fornecidas pelas operadoras do serviço. Assim o presente trabalho pode empregar essa informação para descrever os modelos do sistema e da otimização. Na literatura são encontrados vários trabalhos que consideram o uso de diferentes métodos, entre os mais utilizados estão o Algoritmo Genético - AG e o Recozimento Simulado (Simmulated Annealing - SA). [3], [4], [5], [6], [7], [8]. Alguns trabalhos são orientados ao planejamento e não à otimização de sistemas já existentes. $\mathrm{O}$ presente trabalho pretende otimizar um sistema em funcionamento, com dados reais sobre sua configuração, para maximizar a capacidade disponível para os serviços de voz e dados, minimizando a quantidade de usuários sem cobertura. O parâmetro razão de capacidade $-q$ - oferece uma abordagem interessante sobre o relacionamento entre capacidade e cobertura no padrão WCDMA. Os algoritmos considerados podem encontrar soluções válidas na otimização do parâmetro $q$.

\section{A. Organização do Artigo}

O restante deste trabalho é organizado como apresentado a seguir. Na seção II é feita uma descrição do problema e apresentados o modelo matemático e os requisitos e características da rede UMTS. A função objetivo utilizada e a otimização por Algoritmo Genético e Simmulated Annealing são apresentadas na seção III. Na seção IV são apresentados os resultados obtidos com as simulações do 
sistema. Finalmente, na Seção V são apresentadas algumas conclusões e propostas de trabalhos futuros.

\section{DESCRIÇÃo DO PROBLEMA}

\section{A. Razão de Capacidade em sistemas WCDMA}

Embora o padrão WCDMA aplique os mesmos princípios do padrão CDMA é preciso diferenciar que a capacidade do sistema pode ser limitada no enlace direto (DL) ou reverso (UL) dependendo da configuração do Nó-B, do desempenho da estação móvel (EM) e do perfil de tráfego.

A capacidade de um Nó-B é limitada em DL quando é atingida a máxima potência de transmissão no DL i.e. o Nó$\mathrm{B}$ não tem mais potência de transmissão disponível para satisfazer os requerimentos de novos usuários. Eles poderão ser aceitos somente modificando a configuração do Nó-B. Por exemplo, adicionando novos setores ou novos Nós-B entre outras atividades de planejamento. A proposta apresentada neste trabalho considera um sistema limitado no DL.

Um sistema com carga elevada possui altos níveis de interferência no DL. O pior cenário de interferência no DL acontece se a Potência de Transmissão total $\left(P^{T o t}\right)$ de todos os Nós-B é fixada no valor máximo. A $P^{T o t}$ do Nó-B é compartilhada entre os canais de controle comum $(\mathrm{CPICH}$, $\mathrm{PCPCH}, \mathrm{AICH}, \mathrm{SCH}, \mathrm{PICH})$ e os canais dedicados para tráfego de voz e dados. Entre os canais de controle comum, o CPICH é usado pelas EMs no processo de seleção do Nó$\mathrm{B}$ e estimação da qualidade do canal. $\mathrm{O}$ valor da potência designada aos outros canais de controle é diretamente proporcional ao valor da Potência de Transmissão do CPICH $\left(P_{T X}\right.$ CPICH $)$ [9], e já que a $P^{T o t}$ é constante a capacidade do Nó-B, i.e a potência disponível para os canais dedicados, poderá ser incrementada se o valor da $P_{T X}$ ${ }_{C P I C H}$ for reduzido. Por sua vez, problemas com a cobertura

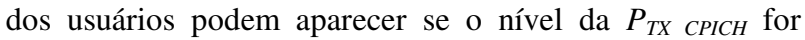
muito fraco. Níveis elevados da $P_{T X}$ СPICH aumentam a interferência no DL e facilitam o aparecimento da poluição do piloto em certas áreas do sistema.

A distribuição dos usuários e a demanda por serviços fazem com que certos Nós-B possuam uma elevada carga de tráfego e, as vezes, pouca potência disponível para os canais dedicados. As conseqüências disso são negativas para a rede: os usuários não podem acessar o sistema (ligações bloqueadas), finalização atípica das ligações e decremento da qualidade do serviço. Esses Nós-B são chamados na literatura de bottleneck cells ou ERBs gargalo [10]. Este problema pode ser combatido, ajustando-se os níveis da $P_{\text {TX CPICH }}$ para balancear a demanda dos usuários entre os Nós-B do sistema, procurando ao mesmo tempo não diminuir o número de usuários com cobertura.

A razão de capacidade $q$ de uma ERB $i$ é definida como a razão entre a potência disponível para tráfego e a demanda total de tráfego dos usuários dento da área de cobertura. Portanto maximizar a capacidade do sistema significa maximizar a razão de capacidade de seus Nós-B.

No modelo de otimização busca-se maximizar o valor médio da $q$ mínima de todos os Nós-B do sistema, minimizando o valor médio da $P_{T X}{ }_{C P I C H}$ e o número de usuários sem cobertura (USC). O modelo é apresentado a seguir.

\section{B. Modelo matemático}

O artigo de Siomina e Yuan [1], define a razão de capacidade entre o Nó-B $i$ e o $\operatorname{bin} j, q_{i j}$ pela expressão

$$
q_{i j}=\frac{P_{i}^{T o t}-\frac{\gamma_{0}}{g_{i j}} I_{i j}}{\sum_{s \in S} \sum_{l \in B(i, j)} \frac{R^{S}}{W} \frac{\gamma^{S} I_{i l}}{g_{i l}} d_{l}^{S}} .
$$

O numerador representa a potência disponível para tráfego, onde:

$P_{i}^{T o t}$ é a potência de transmissão total do Nó-B $i$.

A $P_{T X ~ C P I C H}$ mínima necessária para cobrir o bin $j$ conforme o limiar Ec/Io, é representada em função de $\gamma_{0}, g_{i j}$ e $I_{i j}$.

Sendo: $\gamma_{0}$ o limiar da razão portadora - interferência $\mathrm{Ec} / \mathrm{Io}$; $g_{i j}$ é o ganho de propagação entre o Nó-B $i$ e o $\operatorname{bin} j$, e $I_{i j}$ é a interferência total entre o Nó-B $i$ e o bin $j$.

$\mathrm{O}$ denominador representa a demanda total de tráfego dos usuários dento da área de cobertura, onde:

$R^{S}$ é o valor teórico da conexão do serviço $S$ ou taxa de bits, $W$ é a taxa de Chip,

$\gamma^{S}$ representa o parâmetro Eb/No de cada serviço,

$g_{i l}$ é o ganho de propagação entre o Nó-B $i$ e o bin $l$,

$I_{i l}$, é a interferência total entre o Nó-B $i$ e o $\operatorname{bin} l$, e

$d_{l}^{S}$ é o número de usuários do serviço $S$ no bin $l$.

Na expressão $l \in B(i, j), l$ é o conjunto de bins $j$ que possuem cobertura $B$ do Nó-B $i$.

O ganho de propagação $g_{i j}$, é dado por :

$g_{i j}=-L_{E R B}+G_{A N T}+G_{D I V}+G_{H O}-M_{F}-L_{P}$,

onde:

$L_{E R B}$ representa as perdas no Nó-B (-3 dB),

$G_{A N T}$ é o ganho da antena padrão $(+10 \mathrm{~dB})$,

$G_{D I V}$ é o ganho de diversidade $(+3 \mathrm{~dB})$,

$G_{H O}$ é o ganho de handoff $(+3 \mathrm{~dB})$,

$M_{F}$ é o margem de desvanecimento $(-7,7 \mathrm{~dB})$ e,

$L_{P}$ é a perda de propagação segundo o modelo Cost 231 Hata [10].

A interferência total entre o Nó-B $i$ e o bin $j$, definida na Equação 3, é uma matriz que considera:

- A interferência interna própria da potência total transmitida pelo Nó-B $i$ e recebida no $\operatorname{bin} j$.

- A interferência externa: O bin $j$ recebe também sinais dos outros Nós-B $k$, e

- O ruído térmico do bin.

$I_{i j}=\left(1-\alpha_{j}\right) P_{i}^{T o t} g_{i j}+\sum_{k \in I: k \neq i} P_{k}^{T o t} g_{i j}+v_{j}$.

Onde:

$\alpha_{j}$, é o fator de ortogonalidade, e,

$v_{j}$, é o ruído térmico. 
XXVI SIMPÓSIO BRASILEIRO DE TELECOMUNICAÇÕES - SBrT 2008, 02-05 DE SETEMBRO DE 2008, RIO DE JANEIRO, RJ

Para cada ERB é preciso encontrar o menor valor $q_{i j}$, que representa a menor razão de capacidade (gargalo) que deve ser maximizada. Assim, o problema de otimização pode ser formulado como: encontrar uma combinação de valores de $P_{T X C P I C H}$ e alturas das antenas $\left(h_{E R B}\right)$ que possam garantir a maior cobertura possível, maximizando a razão de capacidade das estações base do sistema.

\section{Requisitos do sistema}

Os parâmetros de funcionamento do sistema estão descritos na Tabela I

TABELA I

REQUISITOS DO SISTEMA

\begin{tabular}{|l|l|}
\hline Freqüência de Operação $[\mathrm{MHz}]-f_{C}$ & 1950 \\
\hline Altura ERB $[\mathrm{m}]-h_{E R B}($ variável) & 10 até 35 \\
\hline Altura EM $[\mathrm{m}]-h_{E M}$ & 1,500 \\
\hline Piso de ruído do bin, $[\mathrm{W}]-v$ & $1,550 \times 10^{-14}$ \\
\hline Fator de ortogonalidade, $\alpha$-tipicamente urbano- & 0,327 \\
\hline Ec/Io do CPICH objetivo & $-18 \mathrm{~dB}$ \\
\hline
\end{tabular}

\section{Características da rede UMTS}

As simulações do sistema foram feitas usando os dados disponibilizados da cidade de Lisboa pelo projeto MOMENTUM [2]. Na Tabela II, se encontram detalhadas as características da rede, da antena e dos parâmetros de potência dos Nós-B [4].

TABELA II

CARACTERÍSTICAS DA REDE UMTS

\begin{tabular}{|l|l|}
\hline \multicolumn{2}{|c|}{ Características da rede } \\
\hline Número de Nodos B & 60 \\
\hline Número de bins & 52500 \\
\hline Tamanho do Bin & $20 \mathrm{~m} \times 20 \mathrm{~m}$ \\
\hline Área total do sistema & $4200 \mathrm{~m} \times 5000 \mathrm{~m}$ \\
\hline \multicolumn{2}{|c|}{ Características da antena } \\
\hline Tipo de antena & Omni-direcional padrão \\
\hline Ganho da antena & $10 \mathrm{dBi}$ \\
\hline \multicolumn{2}{|c|}{ Parâtros de potência } \\
\hline $\begin{array}{l}\text { Potência máxima de transmissão do } \\
\text { Nó-B, } P^{T o t}\end{array}$ & $20 \mathrm{~W}$ \\
\hline $\begin{array}{l}\text { Potência máxima de transmissão do } \\
\text { canal piloto comum, } P_{T X} \text { CPICH }\end{array}$ & $3 \mathrm{~W}$ \\
\hline $\begin{array}{l}\text { Razão da } P_{T X} \text { CPICH frente aos outros } \\
\text { canais comuns no enlace direto. }\end{array}$ & 0.8 \\
\hline $\begin{array}{l}\text { Razão da potência } P_{T X} \text { CPICH } \text { frente à } \\
\text { potencia } P^{T o t}\end{array}$ & 0.1 máximo. \\
\hline Taxa de chip - $W$ & 3.84 Mcps \\
\hline
\end{tabular}

As principais características de cada serviço oferecido pelo padrão UMTS e os valores dos parâmetros mais relevantes de cada um são apresentadas na Tabela III [11].
TABELA III

CARACTERÍSTICAS DOS SERVIÇOS UMTS

\begin{tabular}{|c|c|c|c|}
\hline Classe & Serviço & $\begin{array}{l}\text { Taxa de } \\
\text { bits } R^{S} \\
\text { [Kbps] }\end{array}$ & $\begin{array}{c}\text { Eb/No } \\
\text { objetivo } \\
\text { [dB] }\end{array}$ \\
\hline \multirow{2}{*}{ Conversação } & Telefonia de voz & 12,2 & 5,5 \\
\hline & Vídeo-telefonia & 64 & 3,2 \\
\hline \multirow[b]{2}{*}{ Streaming } & Streaming Multimídia & 64 & 4,2 \\
\hline & $\begin{array}{l}\text { Navegação Web } \\
\text { (WWW) }\end{array}$ & 32 & 3,5 \\
\hline Interativa & $\begin{array}{l}\text { Serviço baseado na } \\
\text { Localização (LBS) }\end{array}$ & 32 & 3,5 \\
\hline \multirow{3}{*}{ Background } & $\begin{array}{l}\text { Serviço de Mensagens } \\
\text { Multimídia (MMS) }\end{array}$ & 32 & 3,5 \\
\hline & $\begin{array}{l}\text { Correio Eletrônico } \\
(E \text {-mail })\end{array}$ & 32 & 3,5 \\
\hline & $\begin{array}{l}\text { Descarga de arquivos } \\
\text { (FDL) }\end{array}$ & 64 & 3,5 \\
\hline
\end{tabular}

O software $\mathrm{MATLAB}_{\circledast}$ foi empregado na programação do modelo matemático e configuração dos algoritmos de otimização.

\section{OTIMIZAÇÃO POR ALGORITMO GENÉTICO E SIMMULATED ANNEALING}

Duas heurísticas de otimização numérica populares encontradas na literatura e que têm dado resultados interessantes são o Algoritmo Genético (AG) e o Recozimento Simulado (Simmulated Annealing - SA). Os métodos procuram as melhores soluções possíveis -ótimaspara o problema dentro de um espaço (conjunto) de potenciais soluções. As soluções são avaliadas por uma função, chamada de função objetivo, que tem o papel de representar o ambiente onde os indivíduos existem.

\section{A. Função Objetivo}

No presente trabalho foram definidas duas funções custo $C_{1}$ e $C_{2}$. A função custo $C_{1}$ representa a média dos valores das $P_{T X C P I C H}$ e é dada por:

$C_{1}=\bar{p}=\frac{\sum_{i=1}^{T o t_{E R B}} p_{T X C P I C H} i}{\operatorname{Tot}_{E R B}}$,

onde $\operatorname{Tot}_{E R B}$ é o número total de Nós-B (ERBs) no sistema. A função custo $C_{2}$ representa a média dos valores das razões de capacidade qij mínimas para cada ERB $i$ e é dada por:

$C_{2}=\bar{q}=\frac{\sum_{i=1}^{\operatorname{Tot}_{E R B}} \min q_{i}}{\text { Tot }_{E R B}}$.

As funções custo $C_{l}$ e $C_{2}$, são agrupadas numa soma ponderada onde os pesos $w_{i}$ dados para cada objetivo foram determinados experimentalmente. $\mathrm{O}$ agrupamento de objetivos, leva em conta também as penalidades e é dado por:

$\min F=\min \left\{\sum_{i=1}^{2} w_{i} C_{i}+\sum_{i=1}^{4}\right.$ Pen $\left._{i}\right\}$. 
XXVI SIMPÓSIO BRASILEIRO DE TELECOMUNICAÇÕES - SBrT 2008, 02-05 DE SETEMBRO DE 2008, RIO DE JANEIRO, RJ

Foram criadas quatro penalidades:

a) Penalização por geração do vetor (interna)

Caso os valores de alturas e potências do vetor avaliado pela função objetivo possuam valores fora dos limiares aceitos na simulação. A penalização 1 é dada por:

Pen $_{1}=0.2$ alt +0.8 pot

alt $=c_{h} / \operatorname{Tot}_{E R B}$, e pot $=c_{p} / \operatorname{Tot}_{E R B}$,

onde $c_{h}$ e $c_{p}$ representam o número de ERBs com $h_{E R B}$ e com $P_{T X ~ C P I C H}$ com valores fora dos limiares estabelecidos nas Tabelas I e II.

b) Penalização por potência CPICH (baixa e alta)

A solução avaliada é penalizada se $\bar{p}$ é maior ou menor que o limiar, $\left(\operatorname{limiar}_{p}\right.$ ) máximo ou mínimo estabelecido para as penalizações 2 e $3\left(P_{e n}\right.$ e $\left.P e n_{3}\right)$ respectivamente no experimento. As funções são exponenciais e estão dadas por:

$\operatorname{Pen}_{2,3}=k_{1}\left(e^{\frac{\Delta}{k_{2}}}-1\right)$,

$\Delta=\left|\bar{p}-\operatorname{limiar}_{p}\right|$

Para $\mathrm{Pen}_{2}$, limiar ${ }_{p}=0,8$ e para $\mathrm{Pen}_{3}, \operatorname{limiar}_{p}=1,2$. Os valores de $k_{1}=50$ e $k_{2}=0,2$ foram obtidos experimentalmente.

\section{c) Penalização por usuários sem cobertura}

Se o número de USC é maior que o limiar determinado para o experimento a função objetivo também é penalizada. A função penalidade $4\left(\mathrm{Pen}_{4}\right)$ é logarítmica e é dada por:

$$
\begin{aligned}
& P_{e n_{4}}=k_{3} \log \left(1+\frac{\Delta}{k_{4}}\right), \\
& \Delta=\left|U S C-\operatorname{limiar}_{U S C}\right|
\end{aligned}
$$

Os valores de $k_{3}=20$ e $k_{4}=3$ foram obtidos experimentalmente.

\section{B. Algoritmo Genético}

Os conceitos sobre o Algoritmo Genético foram tratados no trabalho anterior [12] e são amplamente analisados em [13], sendo descritos no presente trabalho a representação do cromossoma, o método e os operadores utilizados nas simulações realizadas.

\section{a) Representação}

O cromossoma representa as alturas das antenas omnidirecionais e as $P_{T X ~}$ CPICH dos 60 Nós-B do sistema. São portanto, 120 genes que contém um número real cada um entre [10,30] metros para as alturas, e entre [23.304, 34.771] $\mathrm{dBm}$ para as potencias.

\section{b) Método utilizado / Operadores}

O método usado foi o Recipe ou "Receita". Foram usados os operadores Cruzamento - Crossover e Mutação, próprios da ferramenta disponível no $\mathrm{MATLAB}_{\circledast}$, variando os porcentagens de sorteio dos operadores em cada experimento.

\section{Simmulated Annealing}

É um processo inspirado no processo metalúrgico de incremento de temperatura e esfriamento controlados, feito num material para diminuir as impurezas nele. Se o tempo de esfriamento não for o suficiente, as partículas subatômicas do material não se posicionarão adequadamente e o material ainda terá impurezas. Se o tempo de esfriamento é longo demais o material pode esfriar de vez e se tornar inútil. Baseado neste procedimento o algoritmo tenta controlar probabilisticamente o processo de busca, determinando se uma avaliação pior pode ou não ser aceita como uma solução válida. A probabilidade de aceitá-la depende do parâmetro de 'controle de temperatura' $T$ que responde a uma distribuição de Boltzmann, que determina a distribuição de temperatura em sistemas de recozimento. Por sua vez $T$, é diminuído a cada iteração do algoritmo e depende de uma função de esfriamento [10], [14].

\section{a) Representação}

A representação do cromossoma feita para o Algoritmo Genético é valida i.e. a solução possui 120 variáveis que representam a $h_{E R B}$ e a $P_{T X C P I C H}$ dos 60 Nós-B.

\section{b) Método utilizado / Operadores}

Vários experimentos foram realizados com as funções de 'cozimento' e temperatura, obtendo os melhores resultados com a função baseada na distribuição de Boltzmann (@annealingboltz) e com a função de controle de temperatura rápida (@temperaturefast) detalhadas em [15]. Os resultados dos experimentos realizados são apresentados na seção IV.

\section{RESULTADOS}

Foram realizadas simulações do sistema UMTS descrito na Tabela II empregando AG e SA com suas diferentes configurações. Mapas de cobertura do sistema são apresentados considerando uma cobertura referencial inicial (CRI), indicada na Fig. 1 e a cobertura depois da otimização aplicando as duas heurísticas. $\mathrm{O}$ resultado alcançado com o AG é mostrado na Fig. 2.

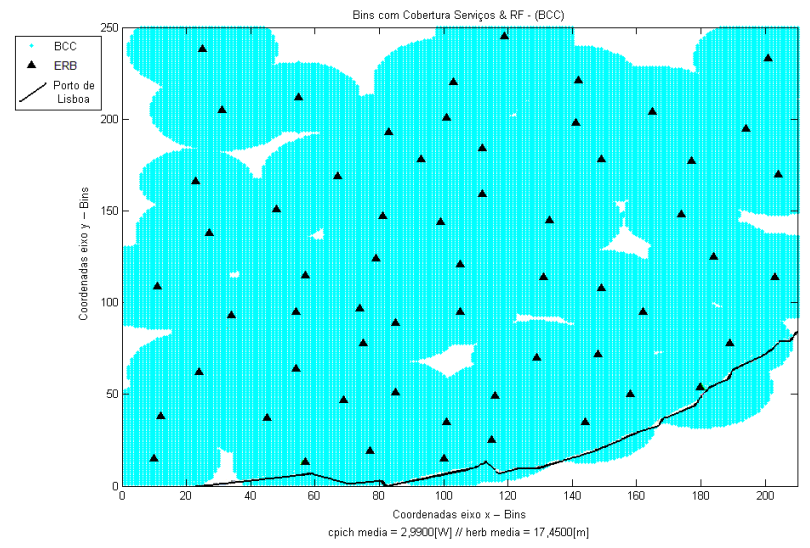

Fig. 1. Cobertura Referencial Inicial para o sistema UMTS de Lisboa. 


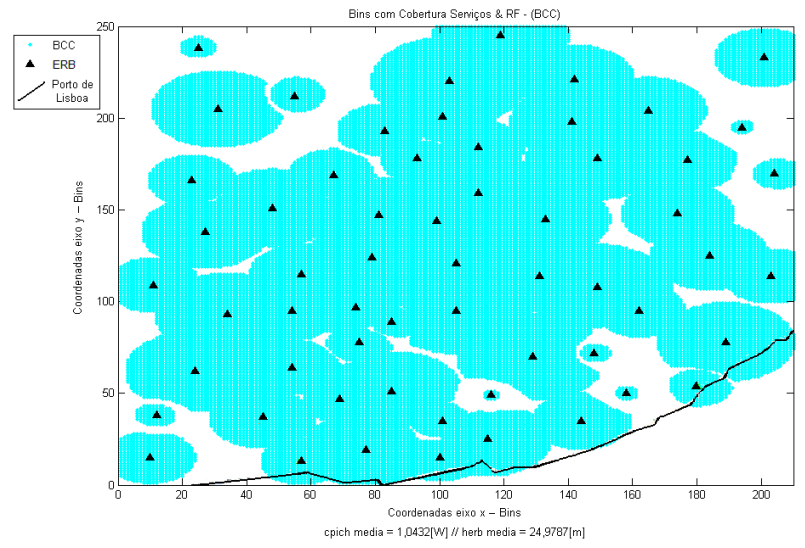

Fig. 2. Mapa de cobertura depois da otimização com AG

A CRI foi obtida considerando as $h_{E R B}$ com seus valores originais e o valor da $P_{T X \text { CPICH }}$ no máximo. Depois de 200 gerações o AG incrementou o valor médio da $h_{E R B} \mathrm{e}$ diminuiu o valor médio da $P_{T X C P I C H}$ do sistema. Já o número de USC atingiu o objetivo do experimento i.e. USC $<200$. Os valores do desempenho do AG são mostrados na Tabela IV. O resultado alcançado com SA é apresentado na Fig. 3.

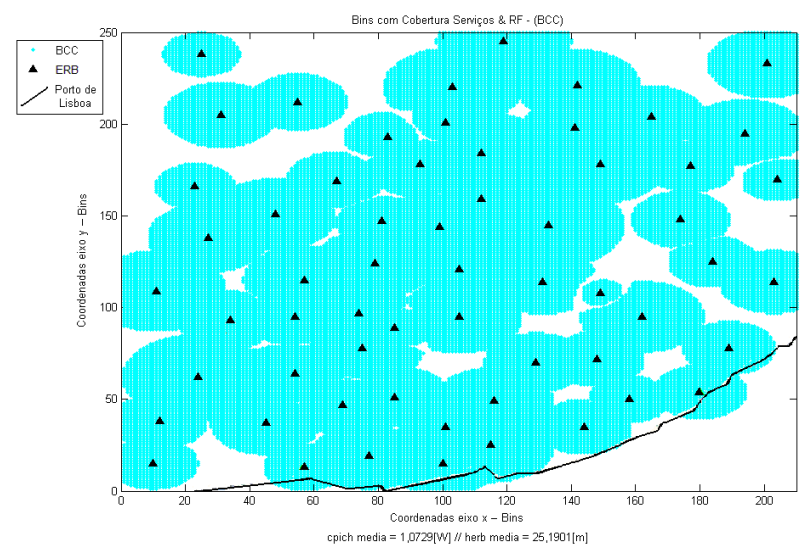

Fig. 3. Mapa de cobertura depois da otimização com SA.

O ponto inicial da busca para o algoritmo SA foi a cobertura referencial inicial. O SA fez 18329 iterações conseguindo os objetivos gerais da otimização. Ou seja, maximizou $q$ média, minimizando $P_{T X \text { CPICH}}$. Os resultados da otimização com SA são apresentados na Tabela V.

TABELA IV

DESEMPENHO DO AG

\begin{tabular}{|l|c|c|}
\hline & $\begin{array}{c}\text { Cobertura } \\
\text { referencial inicial }\end{array}$ & Final Otimização \\
\hline$C_{l}$ & 2,9999 & 1,0432 \\
\hline$C_{2}$ & 0,5591 & 9,9397 \\
\hline$h_{E R B}$ média & 17,4500 & 24,9787 \\
\hline BSC - Bins sem cobertura & 6226 & 14481 \\
\hline USC - Usuários sem cobertura & 71,70 & 199 \\
\hline min $F-$ Valor da F.Objetivo & 404836 & 0,4920 \\
\hline
\end{tabular}

TABELA V

DESEMPENHO DO SA

\begin{tabular}{|l|c|c|}
\hline & $\begin{array}{c}\text { Cobertura } \\
\text { referencial inicial }\end{array}$ & Final Otimização \\
\hline$C_{1}$ & 2,9999 & 1,0729 \\
\hline$C_{2}$ & 0,5591 & 2,5277 \\
\hline$h_{E R B}$ média & 17,4500 & 25,1901 \\
\hline BSC - Bins sem cobertura & 6226 & 12803 \\
\hline USC - Usuários sem cobertura & 71,70 & 179 \\
\hline Min F - Valor da F.Objetivo & 404836 & 8,2016 \\
\hline
\end{tabular}

Nas otimizações com AG e SA, foram considerados os valores de pesos $w_{1}=10$ e $w_{2}=-1$. Valores negativos de $w_{2}$ permitem maximizar $C_{2}$ ( $q$ média) e diminuir o número de USC e BSC. O valor da probabilidade de Crossover $\left(p_{C}\right)$ foi 0,85 , da Mutação $\left(p_{M}\right)$ foi de 0,10 e a população inicial foi 120 indivíduos. Com AS, o valor inicial da temperatura foi de 150 graus "Celsius" e o valor do re-cozimento (reannealing) foi de 130 iterações.

Aparentemente o resultado apresentado nas Tabelas IV e V, faz com que os valores finais de BSC e USC sejam piores no final da otimização, comparados com os valores deles na CRI, i.e. uma redução da cobertura total do sistema. A cobertura inicial permite obter a menor quantidade de USC, embora a penalização por $P_{T X}{ }_{C P I C H}$ elevada faça com que a aptidão da solução a torne indesejável para ser aplicada. No final da otimização, o nível da $P_{T X C P I C H}$ média está em torno de 1,04 a 1,07 W, valores aceitáveis na prática, que indicam que a configuração do AG e do SA produzem resultados finais satisfatórios para o problema apresentado neste trabalho. Com tudo, mesmo com a configuração do SA conseguindo reduzir ainda mais a quantidade de BSC e USC, comparado com o resultado final da tabela IV, a solução proposta pelo AG consegue uma $q$ média quase 4 vezes maior do que a obtida com SA, mantendo quase o

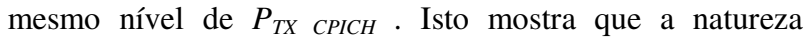
aleatória do AG avalia mais soluções, determinando em cada geração uma solução com melhor aptidão evitando escolher um ótimo local.

\section{CONCLUSÕES}

As técnicas de otimização numérica utilizadas no presente trabalho conseguiram os objetivos propostos: redução significativa da $P_{T X C P I C H}$, aumento significativo da $q$ média e aumento significativo da cobertura para um sistema WCDMA UMTS. O Simmulated Annealing precisa de um ponto inicial i.e. valores iniciais dos parâmetros a otimizar $\left(P_{T X ~ C P I C H}\right.$ e $\left.h_{E R B}\right)$ para assim poder iniciar a procura por uma solução melhor. Isso faz com que a busca por uma solução válida seja demorada ou, o ponto inicial 'dirija' o esforço do algoritmo a encontrar soluções não ótimas ou não válidas. Se não se conhece o valor aproximado da solução desejada, é uma desvantagem rodar o algoritmo SA com um ponto inicial randômico. Já o AG não tem essa limitação, por sua própria natureza probabilística, o que permite focalizar o esforço em encontrar os valores adequados para os parâmetros do $\mathrm{AG}\left(p_{C}, p_{M}\right.$, número de gerações, etc). Foram realizados 22 experimentos com AG e 28 com SA. Os melhores resultados foram apresentados na seção IV. O principal aporte do presente trabalho frente a outros 
trabalhos encontrados na literatura é que não é uma aplicação dos algoritmos no planejamento e sim na otimização de um sistema $3 \mathrm{G}$ já em funcionamento. Ela é baseada num sistema real com dados existentes disponibilizados na internet [2]. A diferença dos trabalhos [16] e [8], onde são comparadas heurísticas de otimização num sistema ideal, neste trabalho foi utilizado um sistema real, onde foi verificado que o AG tem melhor desempenho que o SA. Embora os resultados alcançados mostrem que os algoritmos empregados ajudam efetivamente na melhoria do desempenho do sistema considerado, a implementação sem levar em conta antenas diretivas, Nós-B setorizados, informações específicas do terreno e a mobilidade dos usuários foi um limitante na obtenção de resultados mais adequados com a realidade. Como trabalhos futuros podem ser consideradas outras técnicas como a Busca Tabu ou a Busca Direta na procura de uma solução ótima para o problema apresentado aqui, além da implementação das características adicionais do sistema mencionadas anteriormente.

\section{REFERÊNCIAS BIBLIOGRÁFICAS}

[1] I. Siomina e D. Yuan, Optimization of Pilot Power for Load Balancing in WCDMA Networks. Global Telecommunications Conference, 2004. Globecom'04. IEEE. Vol. 6. Pags: 3872 - 3876.

[2] Projeto MOMENTUM. Informações de redes UMTS disponíveis na internet. http://momentum.zib.de, 2003.

[3] G. Cabral, K. Lage, G. Mateus e R. Franqueira, Planejamento de Redes Celulares de Terceira Geração considerando localização de Estações Radio Base, Controle de Potência e Múltiplos Serviços. Departamento de Ciência da Computação, UFMG, 2003.

[4] I. Siomina, P. Värbrand e D. Yuan, Automated Optimization of Service Coverage and Base Station Antenna Configuration in UMTS Networks. Wireless Communications, IEEE, Vol. 13, Issue 6, Dec. 2006. Pags: $16-25$.

[5] I. Siomina, P-CPICH Power and Antenna Tilt Optimization in UMTS Networks. Proceedings of the Advanced Industrial Conference on Telecommunications, 2005.

[6] I. Siomina e D. Yuan, Pilot Power Management in WCDMA Networks: Coverage Control with respect to Traffic Distribution. Proceedings MSWiM 04, 2004.

[7] I. Siomina, P. Värbrand e D. Yuan, An Effective Optimization Algorithm for Configuring Radio Base Station Antennas in UMTS Networks. IEEE, 2006.

[8] J. Zhang, J. Yang, M. Aydin e J. Wu, Mathematical Modelling and Comparisons of Four Heuristic Optimization Algorithms for WCDMA Radio Network Planning. IEEE Communications Society - ICTON 2006, 2006.

[9] J. Laiho, A. Waker e T. Novosad, Radio network planning and optimization for UMTS. John Wiley \& Sons Ltd., 2006
[10] M. Nawrocki (Editor), Understanding UMTS Radio Network. Modelling, Planning and Automated Optimization. John Wiley \& Sons Ltd., 2006

[11] L. Ferreira (Editor), Final report on traffic estimation and services caracterisation. Momentum Project, 2003

[12] S. Espinosa e P. Nunes, Minimização da Poluição do Piloto em sistemas CDMA aplicando Algoritmo Genético. XXV Simpósio Brasileiro de Telecomunicações - SBrT 07, 2007.

[13] D. Goldberg, Genetic Algorithms in Search, Optimization and Machine Learning. Addison-Wesley Publishing Company, 1989.

[14] A. Martin (Editor), Mathematical Methods for Automatic Optimisation of UMTS Radio Networks. Momentum Project, 2003.

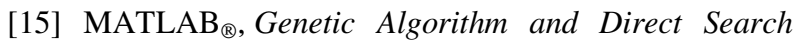
Toolbox Users Guide. The Matworks Inc, 2006.

[16] G. Cabral, K. Lage e G. Mateus, Planejamento de Redes Celulares de Terceira Geração utilizando Algorimos Genéticos e Heurísticas Gulosas. Departamento de Ciência da Computação, UFMG, 2003. 